\title{
Clinical features and surgical outcomes of pulmonary artery sarcoma
}

\author{
Kanhua Yin, MD, ${ }^{\mathrm{a}}$ Zhiqi Zhang, MD, ${ }^{\mathrm{a}}$ Rongkui Luo, MD, ${ }^{\mathrm{b}}$ Yuan Ji, MD, PhD, \\ Difan Zheng, $\mathrm{MD},{ }^{\mathrm{c}}$ Yi Lin, $\mathrm{MD}, \mathrm{PhD},{ }^{\mathrm{a}}$ and Chunsheng Wang, $\mathrm{MD}^{\mathrm{a}}$
}

\section{ABSTRACT}

Objective: Pulmonary artery sarcoma is an exceedingly rare malignancy. There is a lack of consensus regarding its diagnosis and treatment. We reviewed our experience in the surgical management of pulmonary artery sarcoma over an 11-year period.

Methods: From January 2005 to February 2016, 12 patients with pulmonary artery sarcoma (age $51 \pm 14$ years, 5 male) underwent surgical treatment at our center. Nine patients $(75 \%)$ exhibited pulmonary trunk involvement on computed tomography angiography. Six patients $(50 \%)$ were initially misdiagnosed with pulmonary embolism.

Results: Ten patients underwent pulmonary endarterectomy, and 3 of these patients required additional unilateral pneumonectomy. The remaining 2 patients underwent exploratory thoracotomy. One patient $(8.3 \%)$ died in the hospital. The median length of postoperative intensive care unit and hospital stay were 1 day (range, 1-15 days) and 8 days (range, 5-21 days), respectively. The median postoperative survival of the total series was 18 months. Patients who received postoperative combined chemo- and radiotherapy were associated with improved survival compared with those who had isolated adjuvant therapy or surgery alone (median survival 28 vs 8 months, $P=.042$ ).

Conclusions: Although pulmonary artery sarcoma has a very poor prognosis, surgical treatment offers a chance for symptom relief and better long-term outcome. Aggressive postoperative adjuvant treatment may be necessary to improve survival. (J Thorac Cardiovasc Surg 2018;155:1109-15)

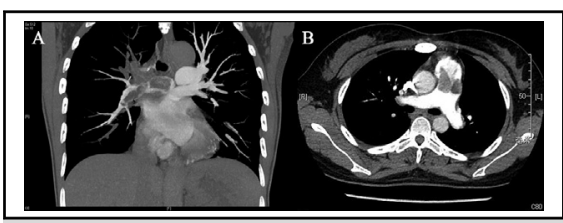

A, Pulmonary artery sarcoma (PAS) invaded the right pulmonary artery (PA) and its distal ends; $B, P A S$ invaded the pulmonary trunk and left PA.

\section{Central Message}

Surgical treatment can relieve symptoms and extend survival for patients with pulmonary artery sarcoma. Combined postoperative chemoand radiotherapy can significantly improve long-term survival.

\section{Perspective}

Pulmonary artery sarcoma presents with nonspecific symptoms and is associated with a high misdiagnosis rate and very poor prognosis. Surgical treatment, including pulmonary endarterectomy and pneumonectomy, offers a chance for long-term survival. Aggressive adjuvant therapies should be considered to improve the postoperative long-term survival.

See Editorial Commentary page 1116.
Primary pulmonary artery sarcoma (PAS) is an exceedingly rare and poorly understood tumor that arises from the intimal layer of the pulmonary artery. ${ }^{1}$ Fewer than 400 cases

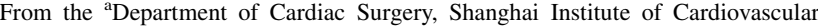
Diseases and ${ }^{\mathrm{b}}$ Department of Pathology, Zhongshan Hospital, Fudan University, Shanghai, China; and ${ }^{c}$ Department of Thoracic Surgery, Fudan University Shanghai Cancer Center, Shanghai, China.

Read at the 12th Annual Academic Surgical Congress, Las Vegas, Nevada, February 7-9, 2017.

This work was funded by the Program of Shanghai Subject Chief Scientist 14XD1401000 and the Fudan University Zhongshan Hospital Youth Research Fund 2016ZSQN24 and 2016ZSQN27.

Drs Yin, Zhang, and Luo are co-first authors who contributed equally to this work.

Received for publication May 26, 2017; revisions received Oct 10, 2017; accepted for publication Oct 27, 2017; available ahead of print Dec 6, 2017.

Address for reprints: Yi Lin, MD, PhD, and Chunsheng Wang, MD, Department of Cardiac Surgery, Shanghai Institute of Cardiovascular Diseases, Zhongshan Hospital, Fudan University, 180 Fenglin Road, Shanghai 200032, China (E-mail: lin. yi@zs-hospital.sh.cn and wangchunshengmd@126.com).

$0022-5223 / \$ 36.00$

Copyright (c) 2017 by The American Association for Thoracic Surgery

https://doi.org/10.1016/j.jtcvs.2017.10.101
}

have been reported since this form of cancer was first described by Mandelstamm in $1923,{ }^{2}$ and the number of case series including more than 10 patients is very limited..$^{3-7}$ Because of the rarity of PAS, the clinical features, diagnosis, and management of this orphan disease remain controversial. This study aimed to review our experience in the management of 12 patients with PAS and evaluate the short- and long-term outcomes.

\section{METHODS}

This study was approved by the Ethics Committee of Fudan University Zhongshan Hospital (approval number: B2016-124). Data were collected

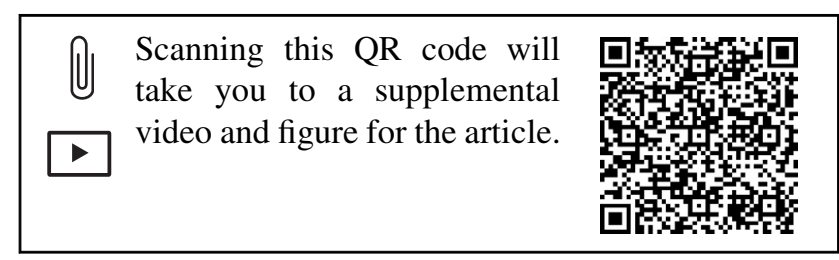




\section{Abbreviations and Acronyms \\ $\mathrm{CPB}=$ cardiopulmonary bypass \\ CTA = computed tomography angiography \\ $\mathrm{CTEPH}=$ chronic thromboembolic pulmonary hypertension \\ ${ }^{18} \mathrm{~F}-\mathrm{FDG}={ }^{18} \mathrm{~F}$-fluorodeoxyglucose \\ PAS = pulmonary artery sarcoma \\ PEA = pulmonary endarterectomy \\ $\mathrm{PET} / \mathrm{CT}=$ positron emission tomography $/$ computed tomography}

retrospectively from patient medical records and the Zhongshan Hospital Electronic Health Record System.

\section{Patient Characteristics}

From January 2005 to February 2016, 12 patients (5 male and 7 female) underwent surgical treatment and were ultimately diagnosed with PAS. The mean age at diagnosis was $51.2 \pm 14.2$ years (range, 21-73 years). Chest pain $(83 \%)$ and asthenia $(58 \%)$ were the 2 most common symptoms, whereas cough, syncope, hemoptysis, headache, and hoarseness were also observed. Eight patients were at New York Heart Association class I or II heart function when admitted, whereas 4 patients were New York Heart Association class III or IV. No patients in this series received preoperative adjuvant therapy.

All patients exhibited varying degrees of pulmonary artery hypertension. The mean pulmonary artery systolic pressure was
$55.9 \pm 14.2 \mathrm{~mm} \mathrm{Hg}$ (range, 33-81 mm Hg). Except for 1 patient who exhibited signs of active bleeding on admission, the other 11 patients underwent contrast enhanced pulmonary artery computed tomography angiography (CTA). Nine patients exhibited pulmonary trunk involvement, and the other 2 had isolated right pulmonary artery neoplasms (Figure 1, $A$ and $B$ ). Five patients underwent ${ }^{18} \mathrm{~F}$-fluorodeoxyglucose $\left({ }^{18} \mathrm{~F}\right.$-FDG) positron emission tomography computed tomography (PET/CT) preoperatively, all of whom showed significant high ${ }^{18}$ F-FDG uptake. Six patients were initially diagnosed with pulmonary embolism, whereas the remaining patients were suspected to have tumors when admitted. The preoperative characteristics of this series are listed in Table 1.

\section{Surgical Techniques}

Pulmonary endarterectomy (PEA) is the preferred procedure in our department. The procedure was initiated via a standard median sternotomy, and cardiopulmonary bypass (CPB) was established via ascending aorta and bicaval cannulations. Aortic crossclamping was performed electively. The pulmonary trunk was sectioned longitudinally, and every effort was made to resect as much of the tumor as possible. Distal embolectomy was performed routinely, but deep hypothermic circulatory arrest was not performed in this series. If the visible tumor invaded the distal pulmonary arteries and thus could not be resected via PEA, a pneumonectomy was performed.

\section{Follow-up and Statistical Analysis}

The follow-up comprised a combination of outpatient visits, telephone calls, and home visits. Overall survival was defined as the interval between the date of the operation and the date of death from any cause or the last follow-up (May 12, 2017).

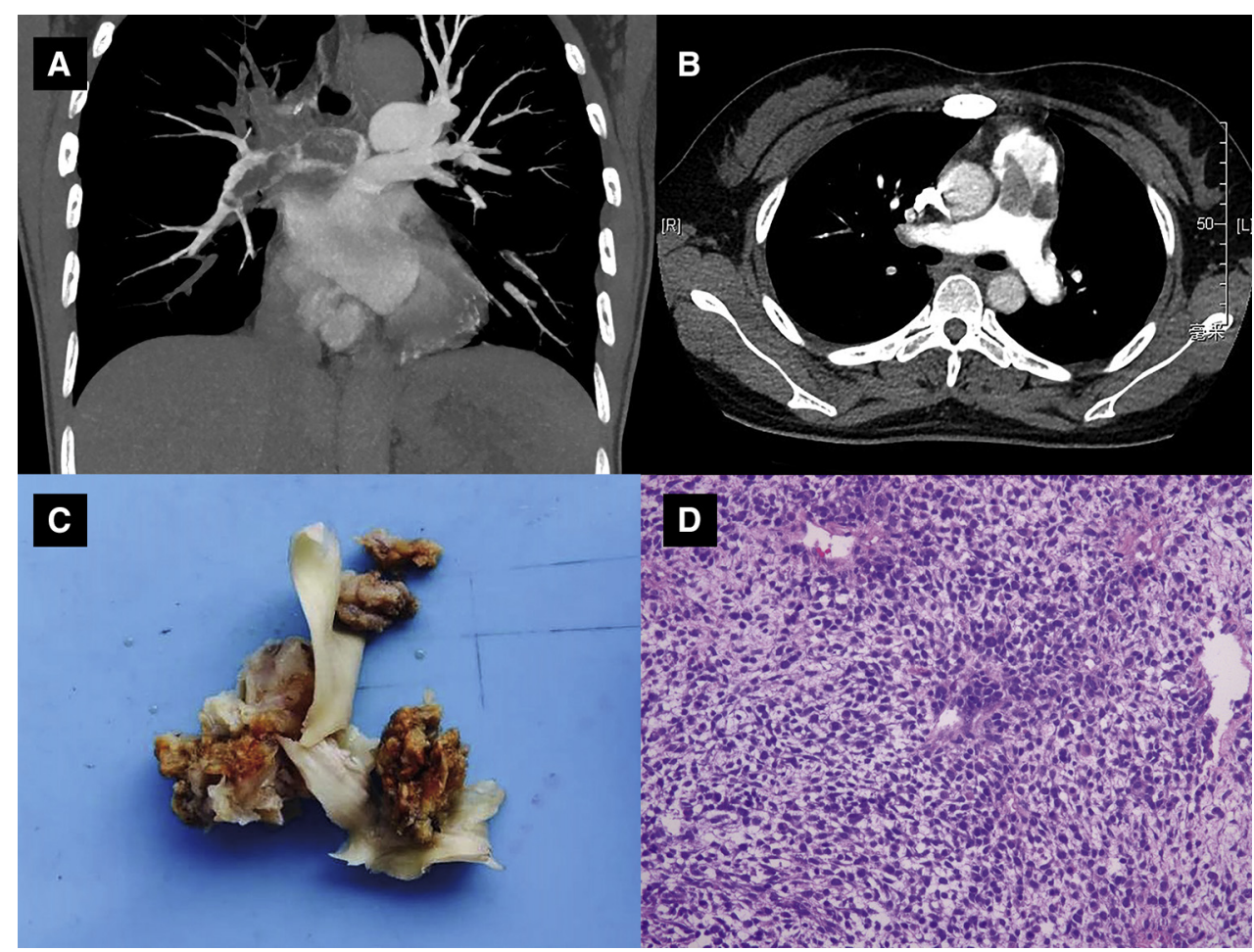

FIGURE 1. The image findings, gross specimen, and histology of PAS. A, Contrast-enhanced pulmonary artery CTA shows a tumor invaded right pulmonary artery and its distal ends; and B, contrast-enhanced pulmonary artery CTA shows a tumor involved pulmonary trunk and left pulmonary artery. C, Gross specimen of a typical patient with PAS: pulmonary artery intimal sarcoma. D, Histology of a typical patient with PAS: rhabdomyosarcoma (hematoxylin and eosin stain; original magnification $\times 200$ ). 
TABLE 1. Patient demographics and preoperative characteristics

\begin{tabular}{lc}
\hline \multicolumn{1}{c}{ Characteristics } & No. or mean \pm SD \\
\hline Sex & 5 \\
Male & 7 \\
Female & $51.2 \pm 14.2(21-73)$ \\
Age, y (range) & $7.7 \pm 9.7(0.25-36)$ \\
Duration from symptoms to surgery, mo (range) & 3.5 \\
$\quad$ Median & \\
Presenting symptoms & 10 \\
Chest pain & 7 \\
Asthenia & 6 \\
Cough & 3 \\
Syncope & 2 \\
Hemoptysis & 2 \\
Headache & 1 \\
Hoarseness & \\
NYHA class & 4 \\
I & 4 \\
II & 3 \\
III & 1 \\
IV & $55.9 \pm 14.2(33-81)$ \\
PASP, mm Hg (range) & \\
Comorbidities & 4 \\
Hypertension & 2 \\
Diabetes mellitus & 1 \\
Previous stroke & \\
Ankylosing spondylitis & \\
Hypothyroidism & \\
\hline SD, Sta & \\
\hline
\end{tabular}

$\overline{S D \text {, Standard deviation; } N Y H A, \text { New York Heart Association; } P A S P \text {, pulmonary artery }}$ systolic pressure.

Continuous data are expressed as the mean \pm standard deviation or medians. Survival analysis was performed via the Kaplan-Meier method and the log-rank test. Surviving patients were censored at the time of last contact. All tests were 2-tailed, and a $P$ value less than .05 was considered statistically significant. The results were analyzed with SPSS for Windows (version 19.0, IBM Corp, Armonk, NY).

\section{RESULTS}

\section{Intraoperative and Pathologic Results}

CPB was initiated in all patients, with the exception of the 2 patients who underwent exploratory thoracotomy, and aortic crossclamping was performed in 7 patients. The mean bypass time and crossclamping time were $83.4 \pm 39.5$ minutes and $41.7 \pm 24.7$ minutes, respectively. Pneumonectomy was necessary in 3 patients ( 2 right and 1 left). One previously reported patient whose tumor involved the pulmonary valve and right ventricle required concomitant right ventricular outflow tract reconstruction with bovine pericardium. ${ }^{8}$ Two patients' tumors were found to be unresectable, as they either encircled the pulmonary trunk and aorta or invaded the pulmonary hilum. These patients underwent exploratory thoracotomy, including 1 emergency procedure.

Five different pathologic tumor types were observed in this series: tumors with myofibroblastic differentiation $(n=6$,
TABLE 2. Operative data and histologic types

\begin{tabular}{lc}
\hline \multicolumn{1}{c}{ Characteristics } & No. or mean \pm SD \\
\hline Surgical techniques & 7 \\
Isolated PEA & 3 \\
PEA $\rightarrow$ pneumonectomy & 2 \\
Exploratory thoracotomy & 10 \\
Cardiopulmonary bypass & $83.4 \pm 39.5(30-137)$ \\
Mean, min (range) & 7 \\
Aortic crossclamping & $41.7 \pm 24.7(15-90)$ \\
Mean, min (range) & 6 \\
Histologic types & 3 \\
Myofibroblastic differentiation & 1 \\
Undifferentiated pleomorphic sarcoma & 1 \\
Leiomyosarcoma & 1 \\
Synovial sarcoma & \\
Rhabdomyosarcoma & \\
\hline
\end{tabular}

SD, Standard deviation; PEA, pulmonary endarterectomy.

$50 \%)$, undifferentiated pleomorphic sarcoma $(\mathrm{n}=3,25 \%)$, leiomyosarcoma $(\mathrm{n}=1,8.3 \%)$, synovial sarcoma $(\mathrm{n}=1$, $8.3 \%)$, and rhabdomyosarcoma $(\mathrm{n}=1,8.3 \%$; Figure $1, C$ and $D$ ). The surgical operative details and histological types of the tumors are summarized in Table 2.

\section{Early Outcomes}

There was 1 in-hospital death (8.3\%): the patient who underwent an emergency exploratory thoracotomy suffered uncontrolled internal bleeding postoperatively. Despite aggressive efforts, the patient died in the intensive care unit on postoperative day 15 . The median lengths of intensive care unit stay and postoperative hospital stay in this series were 1 day (range, 1-15 days) and 8 days (range, 5-21 days), respectively. One patient developed transient hypoxemia but recovered quickly. Another patient developed a bloodstream infection but received timely and appropriate antibiotic therapy, leading to recovery soon thereafter. There were no other severe postoperative complications in this series of patients.

\section{Late Survival}

All 8 late deaths were associated with cancer recurrence at distal pulmonary arteries $(\mathrm{n}=5)$, local recurrence at pulmonary trunk $(\mathrm{n}=1)$, and metastasis $(\mathrm{n}=2)$. One living patient had bilateral lung metastasis during the follow-up. The median postoperative survival of the entire cohort was 18 months. Figure 2 shows the Kaplan-Meier overall survival curve of the entire cohort. There was no significant difference in overall survival between patients with tumor displaying myofibroblastic differentiation and those with other pathologic types $(P=.358$, Figure E1). Four patients $(33 \%)$ received both chemo- and radiotherapy postoperatively, whereas another 3 patients received either chemotherapy or radiotherapy. The remaining 5 patients 


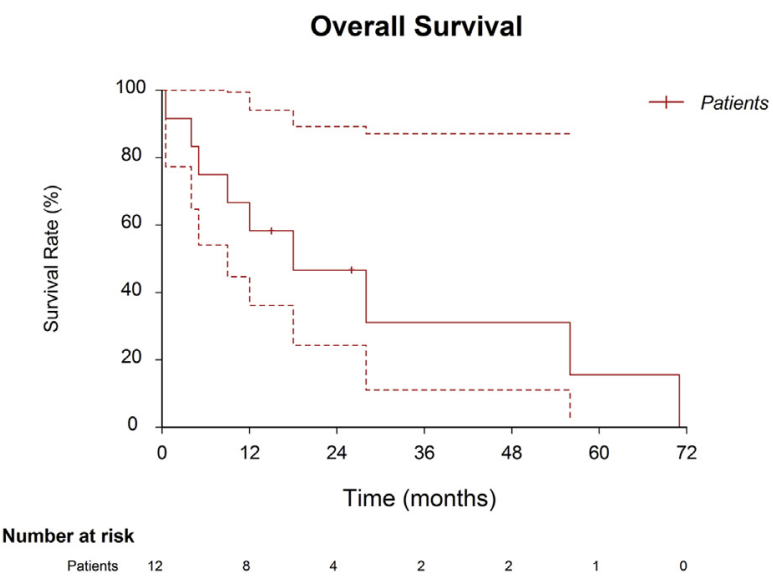

FIGURE 2. Kaplan-Meier overall survival curve: total series. The dotted lines indicate the $95 \%$ confidence interval.

did not receive any adjuvant treatment after surgery. Patients who received postoperative combined chemoand radiotherapy were associated with improved median survival (median survival 28 vs 8 months, $P=.042$, Figure 3 ). Table 3 summarizes the perioperative and long-term surgical outcomes.

\section{DISCUSSION}

\section{Symptoms and Diagnosis}

PAS is considered underdiagnosed because its clinical symptoms are always insidious and its imaging findings are similar to those of chronic thromboembolic pulmonary hypertension (CTEPH). ${ }^{9,10}$ The misdiagnosis may lead to a delay in the correct diagnosis and inappropriate implementation of anticoagulation treatment. Patients usually present with common cardiovascular or pulmonary symptoms, such as chest pain, cough, dyspnea, and syncope. ${ }^{3}$ The presenting symptoms exhibited by the patients in the current study were consistent with previous reports.

\section{Overall Survival}

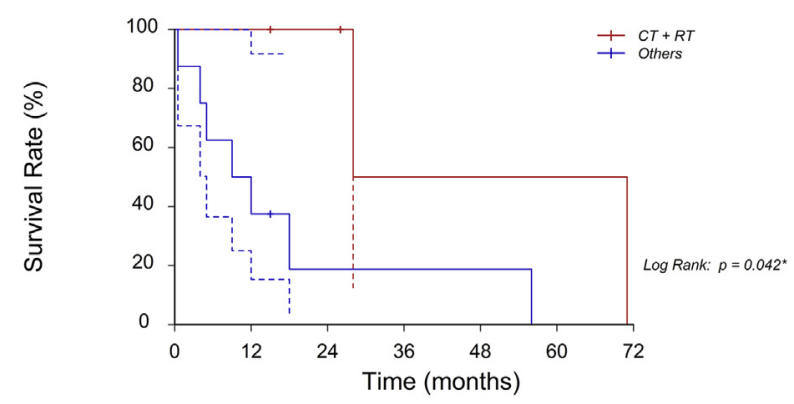

Number at risk

$\begin{array}{rrrrrrrr}\text { CT + RT } & 4 & 4 & 3 & 1 & 1 & 1 & 0 \\ \text { Others } & 8 & 4 & 1 & 1 & 1 & 0 & 0\end{array}$

FIGURE 3. Kaplan-Meier overall survival curves according to postoperative combined adjuvant therapies ( $C T$, chemotherapy; $R T$, radiotherapy). The dotted lines indicate the $95 \%$ confidence intervals.
TABLE 3. Perioperative and long-term outcomes

\begin{tabular}{lc}
\hline \multicolumn{1}{c}{ Characteristics } & No. \\
\hline In-hospital death & 1 \\
Postoperative ICU stay, d, median (range) & $1(1-15)$ \\
Postoperative hospital stay, d, median (range) & $8(5-21)$ \\
Postoperative adjuvant treatments & \\
$\quad$ Chemotherapy + radiotherapy & 4 \\
Chemotherapy alone & 2 \\
$\quad$ Radiotherapy alone & 1 \\
$\quad$ None & 5 \\
Late death & 8 \\
Median survival, mo & 18.0 \\
\hline ICU, Intensive care unit. &
\end{tabular}

Contrast-enhanced CTA is a radiologic test commonly used to evaluate patients suspected of having tumors; this imaging modality can be used to distinguish PAS from CTEPH on the basis of several features, such as low-attenuation filling defects occupying the entire luminal diameter of the proximal or main pulmonary artery, expansion of the involved arteries, and extraluminal tumor extension. ${ }^{11}$ In addition, ${ }^{18}$ F-FDG PET/CT is reportedly useful for differentiating between PAS and CTEPH on the basis of differences in standardized uptake value levels. ${ }^{12,13}$ In this series, all available CTA images demonstrated filling defects. However, definitive imaging diagnoses were difficult to achieve. PET/CT was performed on 5 patients in recent years and demonstrated signs of tumor invasion in all patients. Based on our experience and the findings of previous reports, we suggest that PAS should be suspected in patients with unexplained pulmonary hypertension symptoms who have not responded well to anticoagulation and that ${ }^{18}$ F-FDG PET/CT should be performed as part of a major workup for these patients. An algorithm summarizing our diagnosis and treatment decision-making process is shown in Figure 4.

\section{Surgical Techniques and Outcomes}

PAS is rarely cured by its currently available treatments, and there are no widely accepted treatment protocols to guide the management of this orphan disease. The literature indicates that patient survival time may be as low as 1.5 months without surgical treatment. ${ }^{14}$ Bandyopadhyay and colleagues ${ }^{7}$ reviewed 10 patients with PAS from the Cleveland Clinic and another 381 cases reported in the literature and demonstrated that patients who underwent definitive surgeries had a significantly improved survival compared with partial or no resection. Consequently, surgical resection is still considered the most important option likely to be effective for achieving symptom relief and prolonging survival in patients with PAS.

Various surgical treatments are available for managing PAS, among which PEA and pneumonectomy are 2 most 


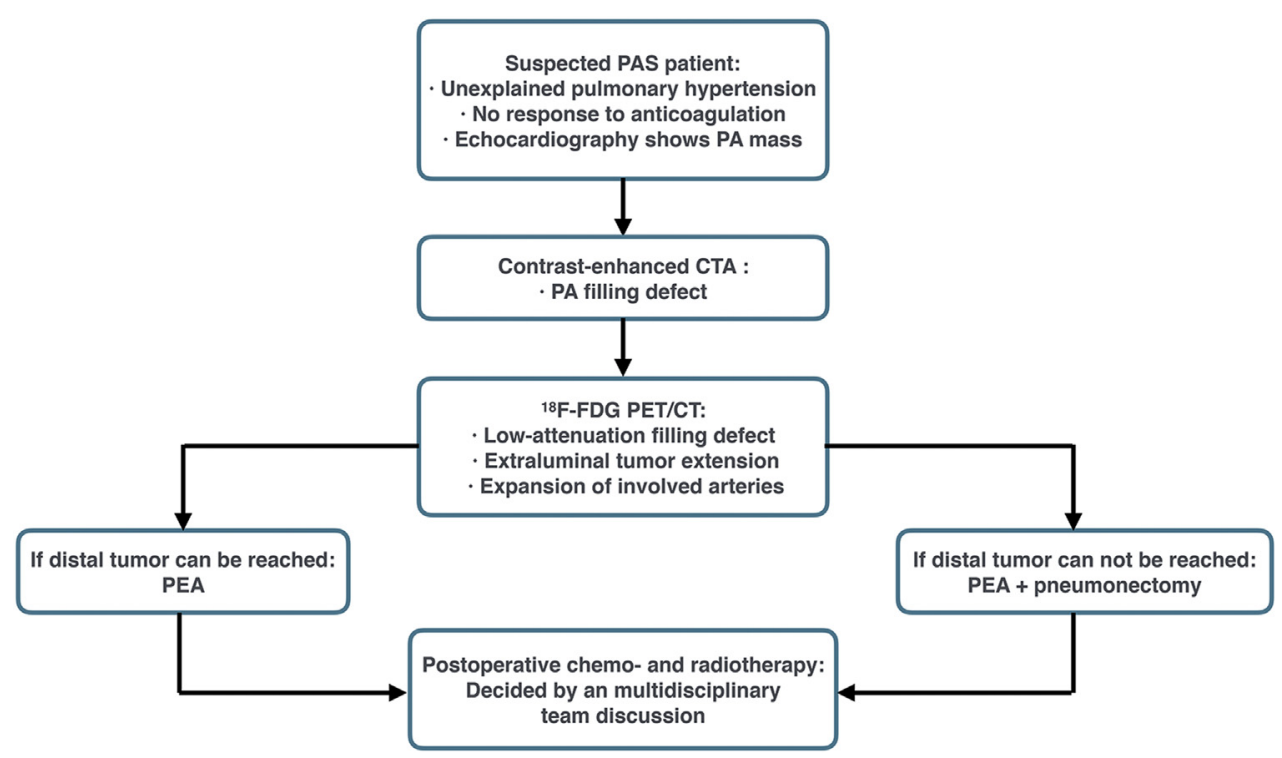

FIGURE 4. Diagnosis and treatment algorithm for patients with suspected PAS. PAS, Pulmonary artery sarcoma; $P A$, pulmonary artery; $C T A$, computed tomography angiography; ${ }^{18} F-F D G,{ }^{18} \mathrm{~F}$-fluorodeoxyglucose; PET/CT, positron emission tomography/computed tomography; $P E A$, pulmonary endarterectomy.

commonly performed procedures. Choosing between these 2 (or more) procedures remains a controversial issue. Grazioli and colleagues ${ }^{6}$ compared PEA and pneumonectomy in 13 patients with PAS, demonstrating that the median survival times were 26.8 months after pneumonectomy and 6.6 months after PEA, respectively. However, Jamieson ${ }^{15}$ believes pneumonectomy may not be an appropriate PAS treatment as it always bilateral or exhibits bilateral seedings. At present, it remains difficult to compare the efficacies of different procedures for managing PAS, not only because of the small number of cases of the disease, but also because of differences in preoperative conditions such as tumor location, tumor extension and preoperative cardiopulmonary function.

Because of the rarity of PAS, studies regarding its outcomes are limited to single-center reports. The largest single center study was performed by Mussot and colleagues, ${ }^{3}$ who treated 31 patients with surgical resection. Twenty-five of these patients underwent PEA, 5 patients underwent pneumonectomy, and 1 patient under right pulmonary artery replacement. The 30-day mortality rate was $13 \%$, and the $1-, 3-$, and 5 -year survival rates were $63 \%, 29 \%$, and $22 \%$, respectively. Bacha and colleagues ${ }^{5}$ reported 23 patients with PAS, of whom 13 underwent lobectomies or biobectomies, 7 underwent radical pneumonectomy or extended radical resection (with CPB support), and another 3 had unresectable tumors. One patient $(4 \%)$ died in the hospital, and the 3-and 5-year survival rate among the patients who underwent resection was $69 \% .^{5}$ In addition to these 2 studies, a few studies, mostly small case series, published across the world in the past 20 years, showed that surgical treatment of PAS can achieve acceptable short-term outcomes; however, the long-term prognosis of this disease remains poor, and the postoperative survival is typically counted in months. ${ }^{4,6,7,16-21}$ Based on the outcomes reported previously, it is fair to conclude that surgery is performed mainly for palliative reasons, with the aim of prolonging survival rather than curing PAS.

Regarding the surgical techniques selected, we routinely performed PEA for patients with PAS and reserved pneumonectomy for patients whose tumors exhibited clear signs of distal invasion and were thus difficult to resect. The advantages of distal embolectomy with CPB were described by Gan and colleagues. ${ }^{21}$ This procedure was also routinely performed in our center. Our in-hospital mortality rate was $8.3 \%(\mathrm{n}=1)$, and the median survival time for the entire series was 18 months; thus, our short- and long-term outcomes were comparable with those of other studies, especially those performed at centers adopting PEA as a major surgical option. ${ }^{4}$ Our findings suggest that PEA is an effective and safe choice for relieving the symptoms of patients with PAS and prolonging their survival.

\section{Pathologic Analysis and Postoperative Adjuvant Therapy}

According to the 2015 World Health Organization classification of tumors, PAS comprises several different histologic types, such as undifferentiated pleomorphic sarcoma, low-grade spindle cell sarcoma with a myxoid background, rhabdomyosarcoma, leiomyosarcoma, undifferentiated sarcoma with epithelioid or round cell features, 


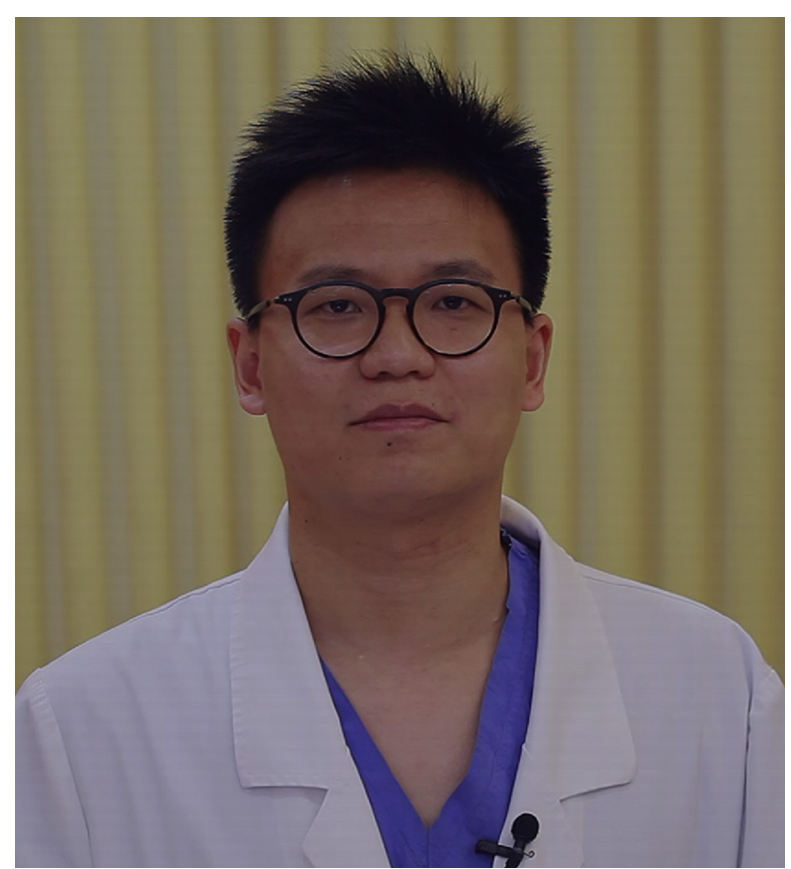

VIDEO 1. Dr Yi Lin, a clinical assistant professor from Zhongshan Hospital of Fudan University and one of principal investigators of this study, introduces the background, methodology, important findings, and conclusion of this paper. Video available at: http://www.jtcvsonline.org/ article/S0022-5223(17)32449-2/fulltext.

synovial sarcoma, epithelioid hemangioendothelioma, and angiosarcoma. ${ }^{22}$ Tavora and colleagues ${ }^{1}$ reported the pathologic results pertaining to a large series of 43 patients with PAS referred from centers throughout the United States. Pleomorphic-fascicular sarcoma was most commonly noted, as it was observed in $65 \%$ of these patients, followed by osteosarcoma (16\%), leiomyosarcoma $(9 \%)$, low-grade myofibroblastic sarcoma $(7 \%)$, and rhabdomyosarcoma $(2 \%)$. From the analysis of Bandyopadhyay and colleagues, ${ }^{7}$ leiomyosarcoma $(80 / 391,20 \%)$ and spindle cell sarcoma $(71 / 391,18 \%)$ were the 2 most common histology types. The distribution of histologic subtypes in the current study was different from that of aforementioned studies, perhaps because of our limited sample size, as $50 \%$ of our patients were found to have tumors with myofibroblastic differentiation.

The effectiveness of postoperative adjuvant therapies remains unclear and a controversial subject. Although most patients who experienced long-term survival received postoperative chemotherapy and/or radiotherapy, few studies have convincingly demonstrated whether adjuvant therapy is helpful for prolonging survival in this particular patient population. Blackmon and colleagues ${ }^{17}$ studied from their 8 PAS patients with 60 patients reported in the literature and found that patients undergoing multimodal treatment experienced significantly better survival outcomes than patients undergoing unimodal treatment (surgical treatment, chemotherapy, or radiotherapy). Similar findings were reported by Wong and colleagues. ${ }^{4}$ However, Mussot and colleagues ${ }^{3}$ observed that there was no difference in survival outcome between patients who did and did not receive postoperative adjuvant therapy in their series. Our study showed that patients who received postoperative combined chemo- and radiotherapy is associated with improved outcomes (median survival 28 vs 8 months, $P=.042$ ). It indicates that for patients with PAS, isolated chemotherapy or radiotherapy may not be enough to extend their life expectancy. Aggressive adjuvant therapy (ie, combined chemo- and radiotherapy) may be required to achieve better long-term survival. However, because of the limited patient volume, further studies, especially study with population data, are warranted (Video 1).

\section{CONCLUSIONS}

PAS is an extremely rare malignancy with a very poor prognosis. The diagnosis mainly relies on symptoms, pulmonary artery CTA, and PET/CT. However, the misdiagnosis rate is still high. Surgical treatment can both relieve the symptoms and extend the life expectance. Postoperative combined chemo- and radiotherapy may be required to achieve a better long-term survival.

\section{Conflict of Interest Statement}

Authors have nothing to disclose with regard to commercial support.

\section{References}

1. Tavora F, Miettinen M, Fanburg-Smith J, Franks TJ, Burke A. Pulmonary artery sarcoma: a histologic and follow-up study with emphasis on a subset of low-grade myofibroblastic sarcomas with a good long-term follow-up. Am J Surg Pathol. 2008;32:1751-61.

2. Mandelstamm M. Über primäre Neubildungen des Herzens [About primary neoplasms of the heart]. Virchows Arch Path Anat. 1923;245:43-54.

3. Mussot S, Ghigna MR, Mercier O, Fabre D, Fadel E, Le Cesne A, et al. Retrospective institutional study of 31 patients treated for pulmonary artery sarcoma. Eur J Cardiothorac Surg. 2013;43:787-93.

4. Wong HH, Gounaris I, McCormack A, Berman M, Davidson D, Horan G, et al. Presentation and management of pulmonary artery sarcoma. Clin Sarcoma Res. 2015;5:3.

5. Bacha EA, Wright CD, Grillo HC, Wain JC, Moncure A, Keel SB, et al. Surgical treatment of primary pulmonary sarcomas. Eur J Cardiothorac Surg. 1999;15: 456-60.

6. Grazioli V, Vistarini N, Morsolini M, Klersy C, Orlandoni G, Dore R, et al. Surgical treatment of primary pulmonary artery sarcoma. J Thorac Cardiovasc Surg. 2014; 148:113-8.

7. Bandyopadhyay D, Panchabhai TS, Bajaj NS, Patil PD, Bunte MC. Primary pulmonary artery sarcoma: a close associate of pulmonary embolism-20-year observational analysis. J Thorac Dis. 2016;8:2592-601.

8. Lu S, Hong T, Wang C. Clinical treatment for pulmonary artery sarcoma. Eur J Cardiothorac Surg. 2010;38:115-6.

9. van Kan C, van der Plas MN, Reesink HJ, van Steenwijk RP, Kloek JJ, Tepaske R, et al. Hemodynamic and ventilatory responses during exercise in chronic thromboembolic disease. J Thorac Cardiovasc Surg. 2016;152:763-71.

10. Jenkins DP, Biederman A, D'Armini AM, Dartevelle PG, Gan HL, Klepetko W, et al. Operability assessment in CTEPH: lessons from the CHEST-1 study. J Thorac Cardiovasc Surg. 2016;152:669-74.

11. Yi CA, Lee KS, Choe YH, Han D, Kwon OJ, Kim S. Computed tomography in pulmonary artery sarcoma: distinguishing features from pulmonary embolic disease. J Comput Assist Tomogr. 2004;28:34-9. 
12. Lee EJ, Moon SH, Choi JY, Lee KS, Choi YS, Choe YS, et al. Usefulness of fluorodeoxyglucose positron emission tomography in malignancy of pulmonary artery mimicking pulmonary embolism. ANZ J Surg. 2013;83:342-7.

13. Ito K, Kubota K, Morooka M, Shida Y, Hasuo K, Endo H, et al. Diagnostic usefulness of 18F-FDG PET/CT in the differentiation of pulmonary artery sarcoma and pulmonary embolism. Ann Nucl Med. 2009;23:671-6.

14. Krüger I, Borowski A, Horst M, de Vivie ER, Theissen P, Gross-Fengels W. Symptoms, diagnosis, and therapy of primary sarcomas of the pulmonary artery. Thorac Cardiovasc Surg. 1990;38:91-5.

15. Jamieson SW. Pulmonary artery sarcoma. Eur J Cardiothorac Surg. 2013;43: 793-4.

16. Kim HK, Choi YS, Kim K, Shim YM, Sung K, Lee YT, et al. Surgical treatment for pulmonary artery sarcoma. Eur J Cardiothorac Surg. 2008;33:712-6.

17. Blackmon SH, Rice DC, Correa AM, Mehran R, Putnam JB, Smythe WR, et al. Management of primary pulmonary artery sarcomas. Ann Thorac Surg. 2009;87: 977-84.
18. Mayer E, Kriegsmann J, Gaumann A, Kauczor HU, Dahm M, Hake U, et al Surgical treatment of pulmonary artery sarcoma. J Thorac Cardiovasc Surg. 2001;121:77-82.

19. Maruo A, Okita Y, Okada K, Yamashita T, Tobe S, Tanimura N. Surgical experience for the pulmonary artery sarcoma. Ann Thorac Surg. 2006;82:2014-6.

20. Chhaya NC, Goodwin AT, Jenkins DP, Pepke-Zaba J, Dunning JJ. Surgical treatment of pulmonary artery sarcoma. J Thorac Cardiovasc Surg. 2006;131:1410-1.

21. Gan HL, Zhang JQ, Zhou QW, Xiao W, Gao YM, Liu S, et al. Surgical treatment of pulmonary artery sarcoma. J Thorac Cardiovasc Surg. 2011;142:1469-72.

22. Travis WD, Brambilla E, Burke AP, Marx A, Nicholson AG. WHO Classification of Tumours of the Lung, Pleura, Thymus and Heart. 4th ed. Lyon: International Agency for Research on Cancer (IARC); 2015.

Key Words: pulmonary artery sarcoma, survival, outcomes, adjuvant therapy 
Overall Survival

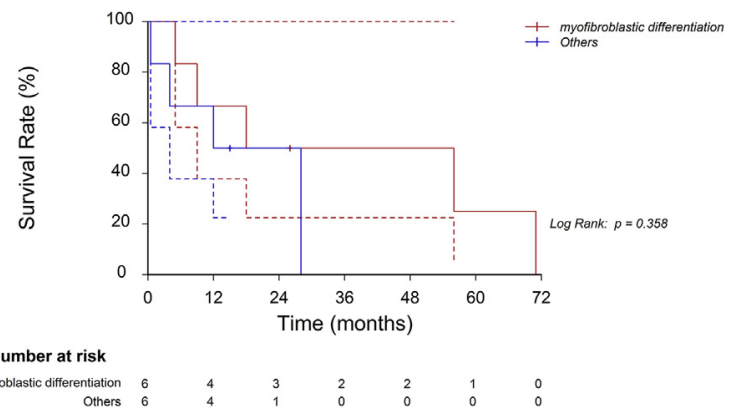

FIGURE E1. Kaplan-Meier overall survival curves according to histologic types. The dotted lines indicate the $95 \%$ confidence intervals. 